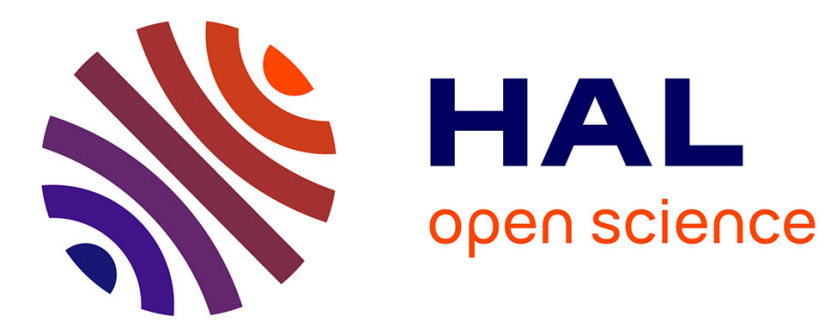

\title{
Backlash fault detection in mechatronic system.
}

\author{
R. Merzouki, Kamal Medjaher, M.A. Djeziri, B. Ould-Bouamama
}

\section{To cite this version:}

R. Merzouki, Kamal Medjaher, M.A. Djeziri, B. Ould-Bouamama. Backlash fault detection in mechatronic system.. Mechatronics, 2007, 17 (6), pp.299-310. 10.1016/j.mechatronics.2007.03.001 . hal00192527

\section{HAL Id: hal-00192527 \\ https://hal.science/hal-00192527}

Submitted on 28 Nov 2007

HAL is a multi-disciplinary open access archive for the deposit and dissemination of scientific research documents, whether they are published or not. The documents may come from teaching and research institutions in France or abroad, or from public or private research centers.
L'archive ouverte pluridisciplinaire HAL, est destinée au dépôt et à la diffusion de documents scientifiques de niveau recherche, publiés ou non, émanant des établissements d'enseignement et de recherche français ou étrangers, des laboratoires publics ou privés. 


\title{
Backlash Fault Detection in Mechatronic System
}

\author{
R. Merzouki ${ }^{a, 1}$, K. Medjaher ${ }^{b}$, M. A. Djeziri ${ }^{c}$ \\ and B. Ould-Bouamama ${ }^{a}$ \\ ${ }^{a}$ LAGIS UMR CNRS 8146, Ecole Polytechnique Universitaire de Lille, Avenue \\ Paul Langevin, 59655 Villeneuve d'Ascq, France. \\ ${ }^{b}$ LAB UMR CNRS 6596, Ecole Nationale Supérieure de Mécanique et des \\ Microtechniques, 26, Chemin de l'Epitaphe, 25030, Besançon, France. \\ ${ }^{c}$ LAGIS UMR CNRS 8146, Ecole Centrale de Lille, Avenue Paul Langevin, 59655 \\ Villeneuve d'Ascq, France.
}

\begin{abstract}
In this paper, a fault detection and isolation model based method for backlash phenomenon is presented. The aim of this contribution is to be able to detect then distinguish the undesirable backlash from the useful one inside an electromechanical test bench. The dynamic model of the real system is derived, using the bond graph approach, motivated by the multi-energy domain of such mechatronic system. The innovation interest of the use of the bond graph tool, resides in the exploitation of one language representation for modelling and monitoring the system with presence of mechanical faults. Fault indicators are deduced from the analytical model and used to detect and isolate undesirable backlash fault, including the physical system. Simulation and experimental tests are done on electromechanical test bench which consists of a DC motor carrying a mechanical load, through a reducer part containing a backlash phenomenon.
\end{abstract}

Key words: Mechatronic system, Backlash, Fault Detection and Isolation, Residuals, Bond graph.

\section{Introduction}

For electromechanical systems, the growth of wear after a long operating period can engender a non neglected backlash. The undesirable presence of back-

1 Corresponding author: Tel. $(+33)$ 328767486, Fax: $(+33) 320337189$.

E-mail address : Rochdi.Merzouki@polytech-lille.fr 
lash acts on the normal operation of the system and can cause considerable damage for the equipment.

Backlash phenomenon is characterized by the existence of dead zone area, which describes the position gap between input and output system positions. Thus, an important dead zone affects the system performance during its control in a closed loop.

In literature, many works have been realized around this topic, and can be classified in three classes: those where the main interest is the control of such systems (e.g. [14], [13]), others are interesting in modelling and identification (e.g. [7], [8]), and for the third class, detection and evaluation of such phenomenon is developed (e.g. [3]). For this last class, two methods of diagnosis exists in the literature: with and without model. Methods without model are based on a training under normal and failing operation (e.g. $[9,15])$, which requires the availability of a well developed fault database for the acquisition of the failing modes. For backlash phenomenon, which describes a mechanical imperfection intern to the system, it is difficult to introduce it from outside the system. Thus, a model based diagnosis method, based on comparison between the nominal model output and the actual operation output is used in this work, where the diagnosis results are strongly dependent on the used model.

Usually, in mechatronic systems, several types of energy are involved. To model this kind of systems, one needs a unified tool like bond graph [10], to represent the involved multiple energy domains. Furthermore, structural properties (observability, invertibility, monitorability, etc.) [2] of bond graph can help in generating fault indicators and fault detection algorithms.

Compared to structural analysis, the use of bond graph allows dealing with one tool from modelling to ARR generation. Whereas, using structural analysis to generate ARRs, one needs first the availability of the constraints list in order to build the bipartite graph and to eliminate the unknown variables by using the incidence matrix. Furthermore, with structural analysis method, the elimination of unknown variables from the constraints is not trivial for complex systems. However, by using bond graphs, it is well known that for an observable system there are as many residuals as number of sensors present on the physical system [4]. So, once the junction equations are written, the elimination procedure is straight forward by using the causal paths from the known to unknown variables on the bond graph model, used in differential causality.

In this work, a contribution on how to detect and to locate the failures introduced by the mechanical backlash is proposed. The main purpose is to distinguish the disturbing backlash from the useful one during a normal operation of an electromechanical system. The useful backlash is defined by the 
initial and non-perturbed dead zone area, necessary for the transient dynamics of the global system. When the dead zone excess its acceptable value, the backlash phenomenon is created and the system will be perturbed.

This paper is organized as follows: after a short description of the electromechanical test bench, a global description of the system modelling is given in Section 3. In Section 4 details on the developed Fault Detection and Isolation (FDI) contribution are presented. Sections 5 and 6 are devoted to simulation and experimental results done on the electromechanical bench, respectively. Finally, a conclusion is given at the end of this paper.

\section{Electromechanical actuator description}

The test bench presented in Fig. 1 has been developed to identify some mechanical imperfections such as: friction, backlash and flexion. It was developed according to a specifications relating on continuous modeling of backlash, flexibility and friction torques [7]. It describes an electromechanical system made up of a motor reducer involving an external load. The motor part is actuated by a DC motor delivering a relative important mass torque. This test bench contains mechanical imperfections such as: friction, backlash and elasticity within the transmission system. Its advantage is that one can vary the amplitudes of the mechanical imperfections located in the load part (Fig. 1). Coulomb friction is represented by a contact of various components of the system with different rigidities. Viscous friction depends on the viscosity of the lubricant contained between surfaces in contact. Backlash phenomenon is represented by two independent mechanical parts, whose transmission is carried out via a dead zone varying between 0 and 24 degrees. A spring system is placed between the two mechanical parts in order to deliver a smooth transmission.

On this test bench, one can measure the input and output positions of the reducer part by using two incremental encoders (Fig. 1).

\section{Test bench global model}

The electromechanical system of Fig. (1) can be considered as a concatenation of four parts: electrical part of DC motor, mechanical part of DC motor, reducer part and load part (Fig. 2). In the following modelling, the static friction effects are not considered for mechanical aspect. Only a viscous friction, backlash phenomenon and flexibility of the transmission links are taken into account as a mechanical imperfections. 


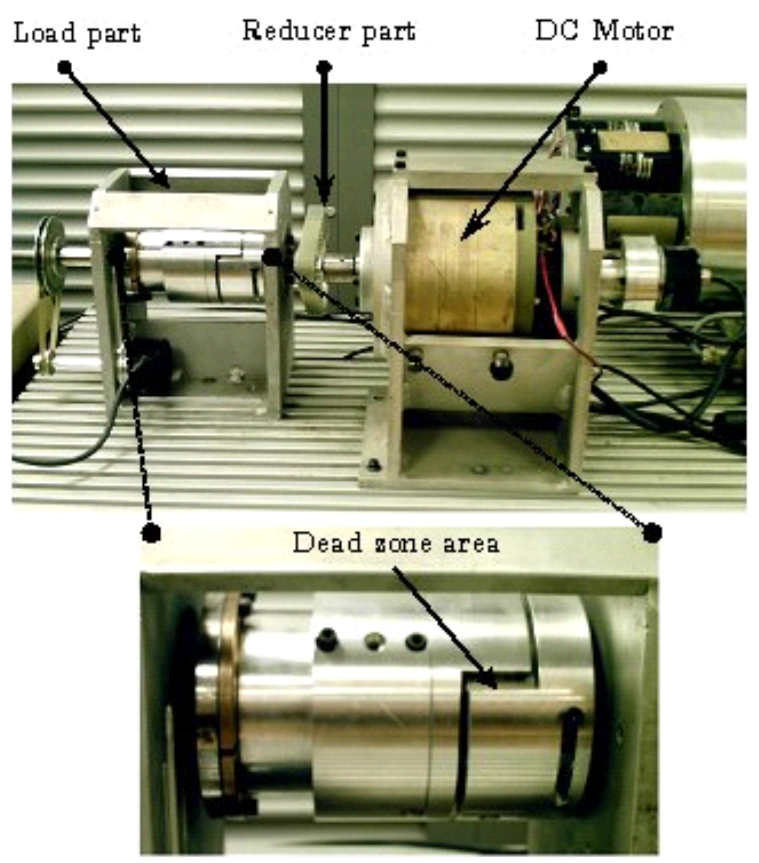

Fig. 1. Electromechanical system.

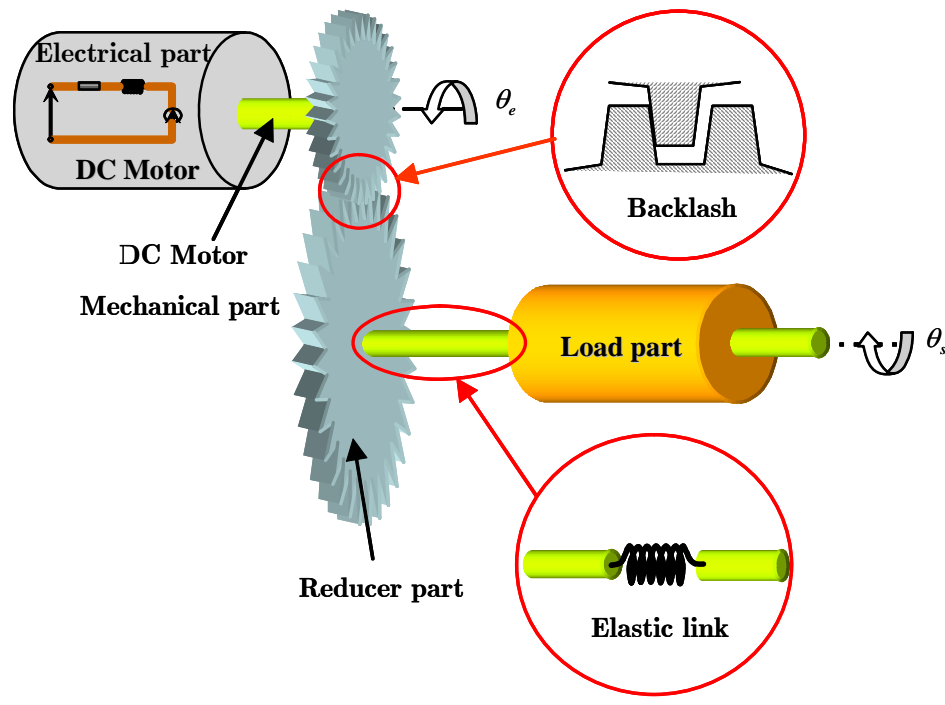

Fig. 2. Electromechanical system parts.

\subsection{Word bond graph}

The word bond graph represents the technological level of the model where the global system is decomposed into four subsystems (Fig. 3). Compared to classical block diagram, in this representation, the input and output of each subsystem are defined by power variables represented by a conjugated pair of effort-flow $(e, f)$. The power variables used for the studied system are: 


$$
\begin{aligned}
& (\text { Torque, Angular velocity })=\left\{\left(\Gamma, \dot{\theta}_{e}\right),\left(C, \dot{\theta}_{e}\right),\left(N . C, \dot{\theta}_{s}\right)\right\}, \\
& (\text { Voltage, current })=\left(U_{0}, i\right) .
\end{aligned}
$$

These true bond graph variables are associated respectively with electrical and mechanical rotation aspects of the physical system.

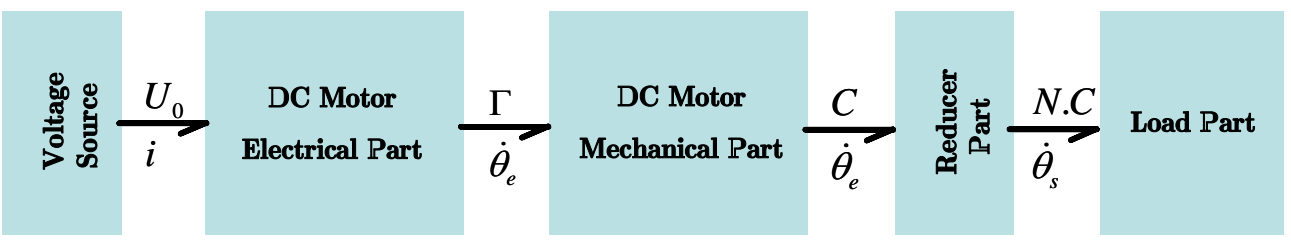

Fig. 3. Word bond graph of the electromechanical system.

\subsection{Bond graph model}

In this subsection, dynamic bond graph model, in preferred integral causality, of each subsystem described in Fig. 2 is presented.

\subsubsection{Electrical part of the DC motor}

This part is represented by an electrical circuit (Fig. 4) composed by: an input voltage source $U_{0}$, resistance element $R_{e}$, inductance $L$ and back-electromotive force, which is linearly dependent on the angular velocity $\dot{\theta}_{e}$ of the rotor and is equal to $k_{e} \cdot \dot{\theta}_{e}$ where $k_{e}$ is an electrical constant.

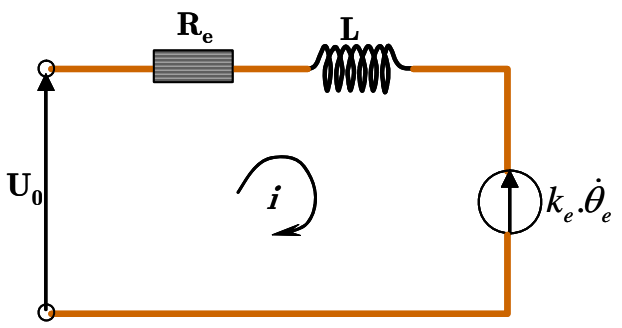

Fig. 4. RLC electrical circuit

The corresponding bond graph model in preferred integral causality of the electrical part is given in Fig. 5 .

Let's note $e_{1}, P_{1}$, and $L$, effort, momentum and algebraic value of bond graph element $I ; f_{4}$ flow variable of link 4 and $S e: U_{0}$ the input voltage source, then the following state equation can be derived: 


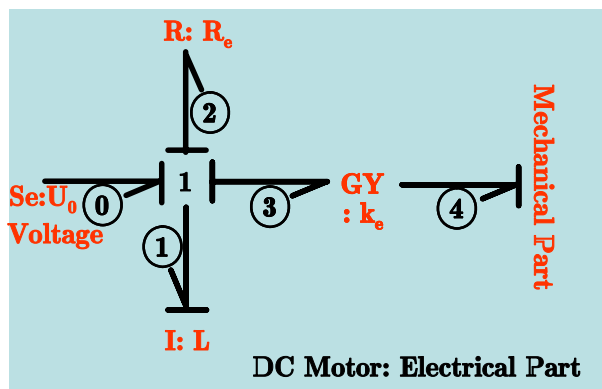

Fig. 5. Bond graph model of electrical part.

$$
e_{1}=\frac{d P_{1}}{d t}=-R_{e} \cdot \frac{P_{1}}{L}-k_{e} \cdot f_{4}+U_{0}
$$

By replacing the effort and flow bond graph variables by their corresponding electrical ones of Fig. 4, Eq. (1) can be written as:

$$
L \cdot \frac{d i}{d t}=-R_{e} \cdot i-k_{e} \cdot \dot{\theta}_{e}+U_{0}
$$

The gyrator $(G Y)$ element on the bond graph model represents the power transfer from electrical to mechanical part.

\subsubsection{Mechanical part of the DC motor}

This subsection describes the mechanical part of the $D C$ motor represented by its inertia $J_{e}$, viscous friction coefficient $f_{e}$, transmission axis elasticity $K$ and a motorized torque $U$. In this part, the influence of backlash phenomenon is expressed by a disturbing torque $w$, and represented by a modulated effort source in the bond graph model. The corresponding bond graph model of the mechanical part is given by Fig. 6 .

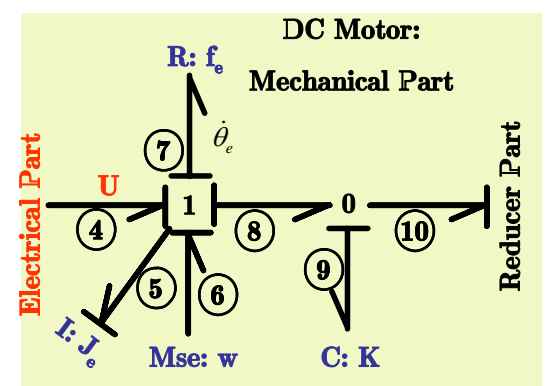

Fig. 6. Bond graph model of mechanical part.

In order to understand the role of the modulated effort source (Mse $: \omega)$, it is worth explaining the backlash mechanism inside the electromechanical system. 
Backlash mechanism is constituted of two mechanical and unconnected bodies (Fig. 7), where 'Body 1' tries to transmit motion to 'Body 2' via a dead zone of amplitude 2. $j_{0}$ ( $j_{0}$ is dead zone magnitude). The transmission will be correct when the two bodies are in contact (i.e. bodies positions are identical). Otherwise (i.e. bodies are not contacted), the transmission will be delayed due to the presence of a dead zone. The analytical relation between the bodies positions is described by a hysteresis behavior relation. However, in order to avoid discontinuous disturbances due to a rigid contact, a smooth contact area has been realized for the test bench. This is used to deaden the shock of the contact and is modelled by a spring system of rigidity $K$ (Fig. 7 ).

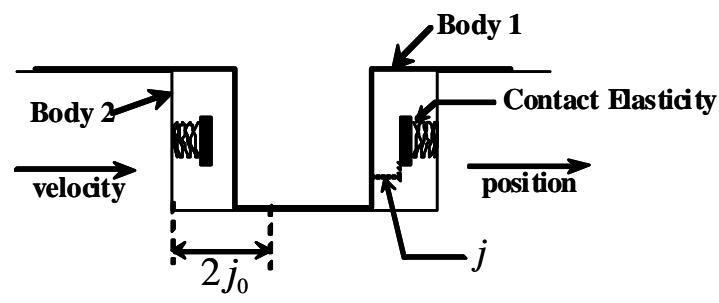

Fig. 7. Backlash mechanism.

Thus, a smooth and continuous model of transmitted torque $w$, which is developed in [6] and illustrated in Fig. 8, is chosen to be identified and used as a modulated effort source (Mse $: w)$. This latter includes a sigmoid function expressed by the following relation:

$$
w=-4 . K \cdot j_{0} \cdot \frac{1-e^{-\gamma \cdot z}}{1+e^{-\gamma \cdot z}}
$$

where $z$ is the difference between input and output reducer positions and $\gamma$ the slope constant.

By adding the disturbing torque $w$, Eq. (3) to the linear transmitted torque $C_{0}$, which describes a flexible link given by:

$$
C_{0}=K . z,
$$

the approximate continuous transmitted torque $C$ is obtained as follows:

$$
C=K .\left(z-4 . j_{0} \cdot \frac{1-e^{-\gamma \cdot z}}{1+e^{-\gamma \cdot z}}\right)
$$

Since the real backlash amplitude $j_{0}$ is equal to a constant, its time variation is null. The disturbing torque $w$ has a sigmoid function form, characterized by its decreasing slope constant $\gamma$. The slope $\gamma$ is chosen to give the best approximation of the transmitted torque inside the dead zone defined within the interval $\left[-j_{0},+j_{0}\right]$ of Fig. 8 . 


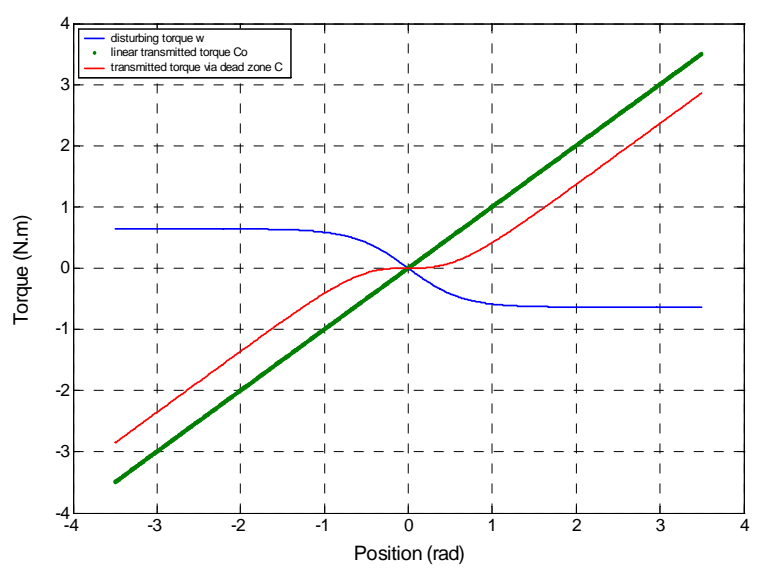

Fig. 8. Approximation of transmitted torques.

Let's note $e_{5}, P_{5}, J_{e}$, effort, momentum and algebraic value of element $I, f_{4}$ flow variable of link $4, f_{9}, K$ flow and algebraic value of bond graph element $C$, and $M S e: w$ the modulated effort source. Thus, the following state equation can be derived:

$$
e_{5}=\frac{d P_{5}}{d t}=-R_{7} \cdot \frac{P_{5}}{J_{e}}+k_{e} \cdot f_{4}+w-K \cdot \int f_{9} \cdot d t
$$

By replacing the bond graph variables by their corresponding mechanical and electrical ones, Eq. (6) becomes:

$$
J_{e} . \ddot{\theta}_{e}=-f_{e} \cdot \dot{\theta}_{e}+k_{e} . i+w-K . z
$$

\subsubsection{Reducer part}

This part deals with the mechanical gears which link the mechanical and the load parts with a reduction constant $N$. Bond graph model of this physical subsystem is given by Fig. 9 where the reducer is represented by a transformer element $T F$ between the motor axis (with velocity $\dot{\theta}_{e}$ ) and the load (with velocity $\left.\dot{\theta}_{s}\right)$.

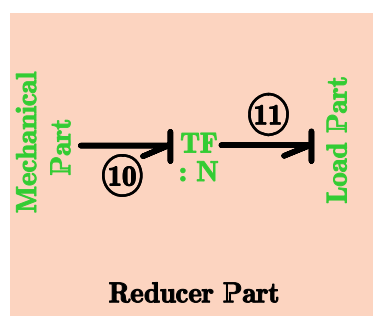

Fig. 9. Reducer part bond graph model. 
The constitutive relation of the bond graph element $T F$, of Fig. 9, is written as:

$$
f_{10}=N \cdot f_{11}
$$

where $f_{10}$ and $f_{11}$ are the corresponding flow variables of links 10 and 11 . In mechanical domain, Eq. (8) becomes:

$$
\dot{\theta}_{e}=N . \dot{\theta}_{s}
$$

\subsubsection{Load part}

This part describes the load part of the electromechanical system. It is represented by its inertia $J_{s}$, viscous friction coefficient $f_{s}$ and backlash disturbing torque N.w. Its corresponding bond graph model is given by Fig. 10 .

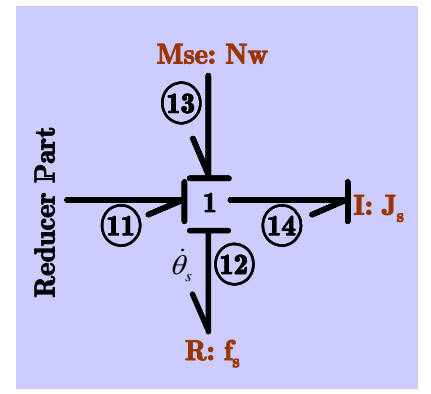

Fig. 10. Load part bond graph model.

Let's note $e_{14}, P_{14}, J_{s}$, effort, momentum and algebraic value of element $I, f_{9}$, $K$ flow and algebraic value of bond graph element $C$ (Fig. 6); and MSe : N.w the modulated effort source. The following relation can then be deduced from the 1 junction of Fig. (10):

$$
e_{14}=-R_{12} \cdot \frac{P_{14}}{J_{s}}+S e_{13}+N \cdot K \cdot \int f_{9} \cdot d t .
$$

After replacing the bond graph variables of Eq. (10) by their corresponding mechanical ones, one can get:

$$
J_{s} . \ddot{\theta}_{s}=-f_{s} . \dot{\theta}_{s}+N . w+N . K . z .
$$

\subsubsection{Electromechanical global bond graph model}

A concatenation of the bond graph models of the different parts described above leads to the global bond graph model of the electromechanical system 
given in Fig. 11.

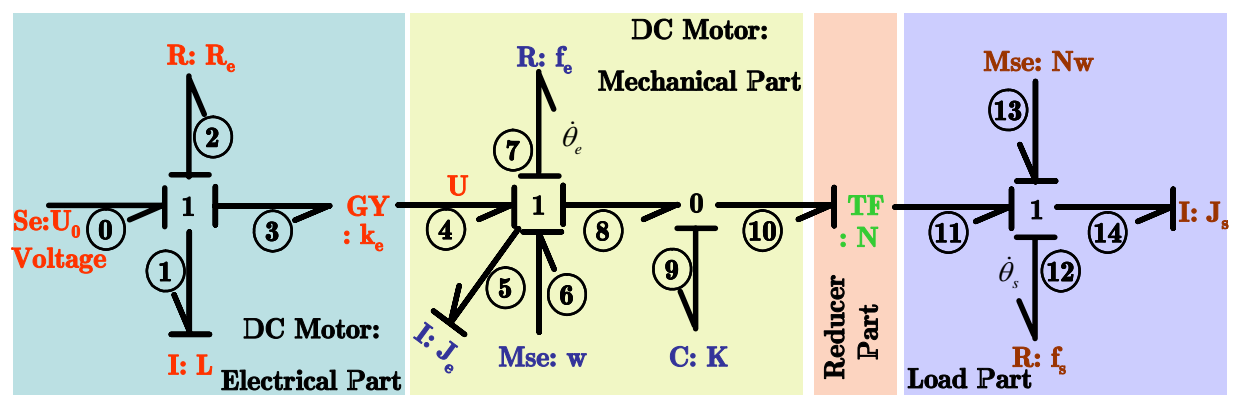

Fig. 11. Global bond graph model of the electromechanical system.

The dynamic and nonlinear model of the test bench, including the backlash, deduced from the global bond graph model is given by the following relations:

$$
\left\{\begin{array}{l}
L . \frac{d i}{d t}+R_{e} \cdot i+k_{e} \cdot \dot{\theta}_{e}=U_{0}, \\
J_{e} \cdot \ddot{\theta}_{e}+f_{e} \cdot \dot{\theta}_{e}+w+K . z=\Gamma, \\
J_{s} \cdot \ddot{\theta}_{s}+f_{s} \cdot \dot{\theta}_{s}=N . w+N . K . z, \\
\dot{z}=\dot{\theta}_{e}-N . \dot{\theta}_{s} ;
\end{array}\right.
$$

where $\Gamma=k_{e} . i$, is the input motorized torque.

Fig. 12 represents the correspondence between the bond graph models and the physical system parts. This is done to show the location of undesired backlash imperfection.

\section{Simulation tests}

\subsection{Software Implementation}

Simulation step is done on a specific bond graph software SYMBOLS 2000 [12], which is an object oriented hierarchical modelling software. It allows users to create models using bond graph, block-diagram and equation models. Differential causalities and algebraic loops are solved out using its powerful symbolic solution engine. Nonlinearities and user code can be integrated in single editing IDE (Integrated Development Environment). The iconic modelling facility allows system-morphic model layout. It also has many post-processing facilities over the simulated result. Thanks to a developed generic item database which consists of a set of predefined models, and has been incorporated 


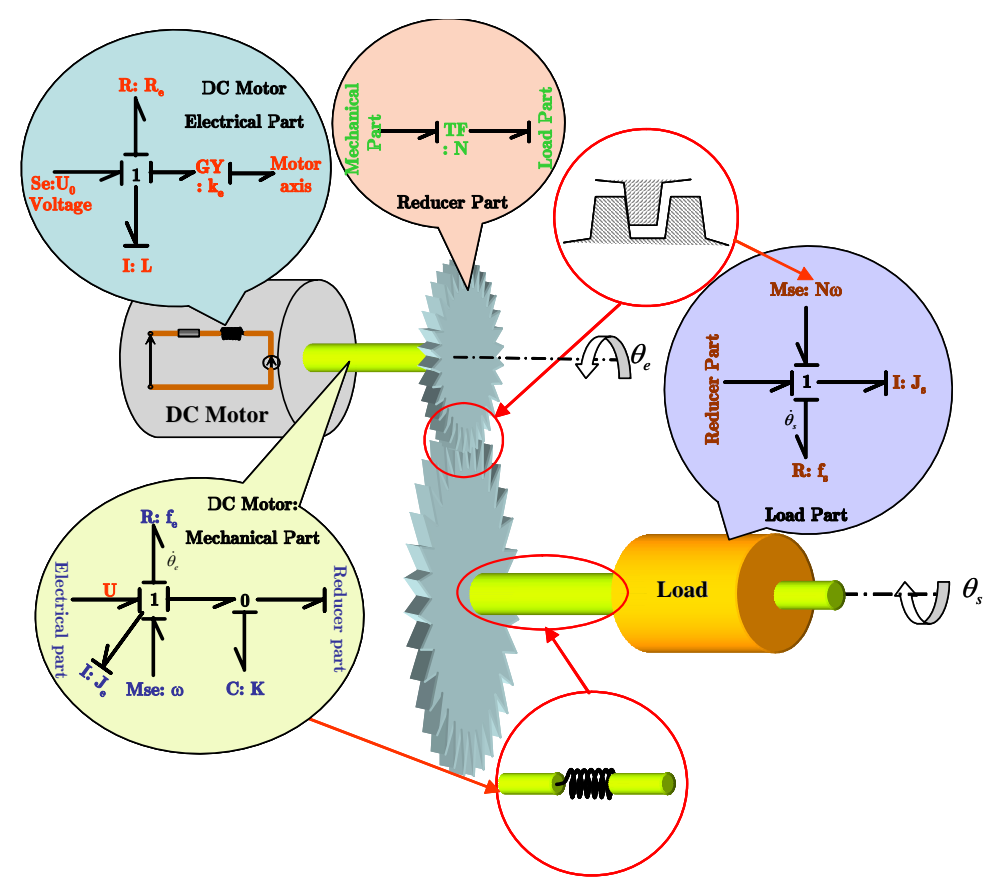

Fig. 12. Correspondence bond graph - electromechanical system.

as capsules in the software $S Y M B O L S 2000$, the designer can easily build the dynamic models of several physical systems from the Process and Instrumentation Diagram $(P \& I D)$ by just connecting different sub models. The global dynamic in symbolic format is obtained connecting different icons. Behind each submodel the bond graph model is hinted (Fig. 13). If parameter values are available, the model can be simulated using own Symbols function or Matlab, S-Function

\subsection{Simulation results}

Simulation parameters used for the electromechanical system are given in table (1).

For an input voltage signal like that one of Fig. 14, one can obtain the graphical characteristic of the backlash disturbing torque of Fig. 15 and the global transmitted torque to the load of Fig. 16. This latter shows a dead zone of magnitude $2 . j_{0}=0,4 \mathrm{rad}$ and a linear transmitted torque outside the dead zone. 


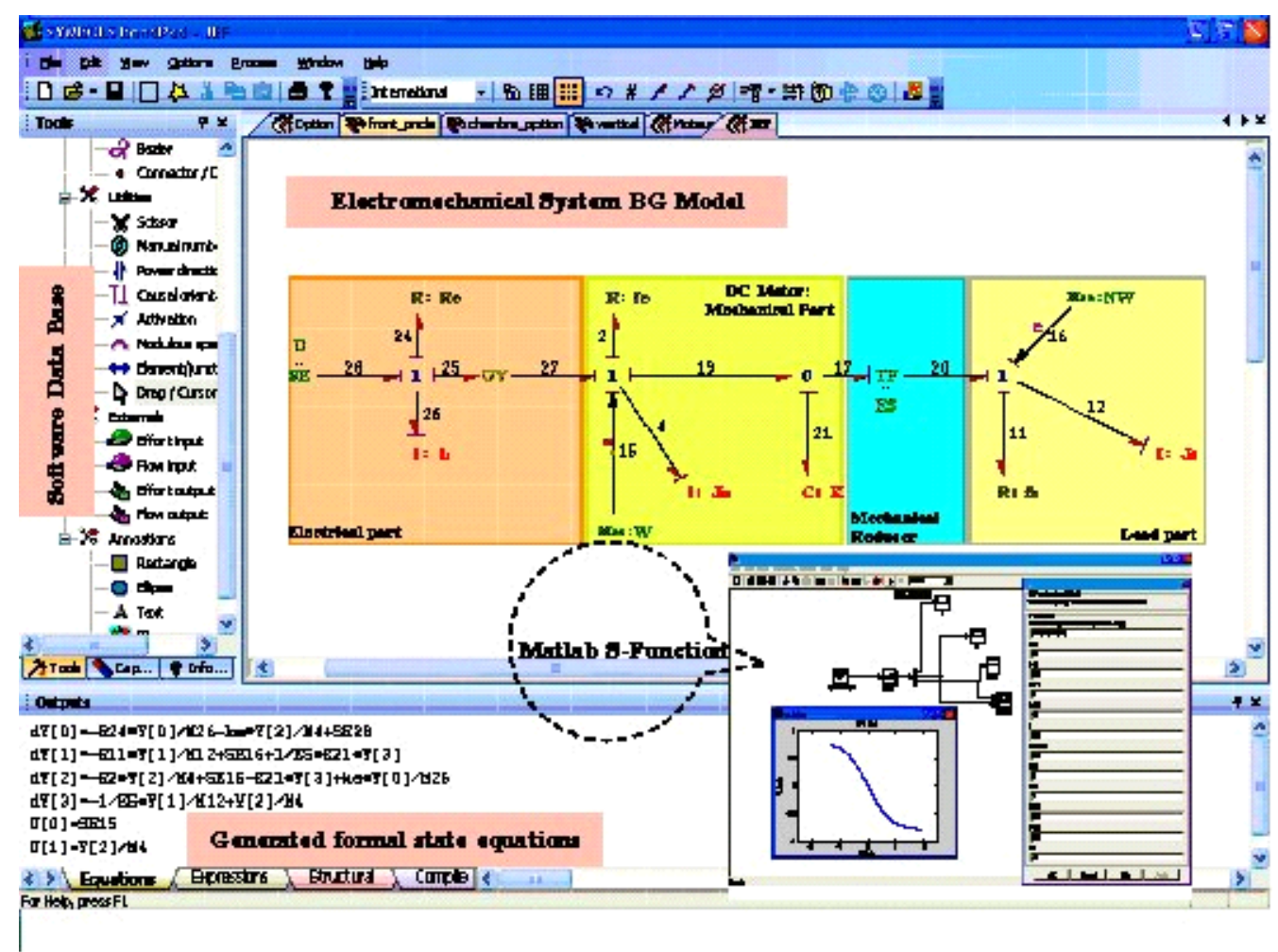

Fig. 13. Softaware interface using SYMBOLS 2000 and Matlab-Simulink

Table 1

\begin{tabular}{|l|l||l|l|}
\hline Parameter & Value & Parameter & Value \\
\hline$J_{e}$ & $0.8\left(\mathrm{Kg} \cdot \mathrm{m}^{2}\right)$ & $K$ & $1(\mathrm{~N} \cdot \mathrm{m} / \mathrm{rad})$ \\
\hline$J_{s}$ & $10\left(\mathrm{Kg} \cdot \mathrm{m}^{2}\right)$ & $k_{e}$ & $0.5(\mathrm{~N} . \mathrm{m} / \mathrm{A})$ \\
\hline$f_{e}$ & $0.1(\mathrm{~N} \cdot \mathrm{m} \cdot \mathrm{sec} / \mathrm{rad})$ & $j_{0}$ & $0.2(\mathrm{rad})$ \\
\hline$f_{s}$ & $8($ N.m. sec $/ \mathrm{rad})$ & $\gamma$ & $2.5\left(\mathrm{rad}^{-1}\right)$ \\
\hline$L$ & $0.5(\mathrm{H})$ & $N$ & 5 \\
\hline$R_{e}$ & $0.3(\Omega)$ & & \\
\hline
\end{tabular}

Simulation model parameters.

\section{Backlash torque detection}

In this part, a model based Fault Detection and Isolation (FDI) approach $[4,11,5]$ is used in order to detect a undesirable dead zone and disturbing backlash torque in an electromechanical system in presence of wear phenomenon. This detection can help on improving the system performances by developing adaptive control laws [7].

By using the FDI algorithms proposed in [4,1], a list of Analytical Redundancy 


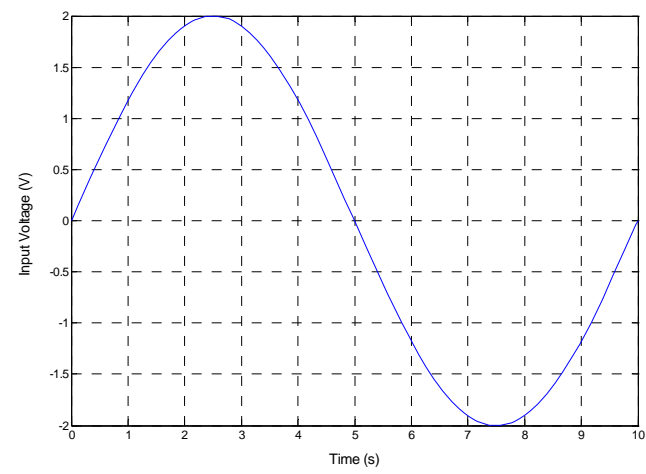

Fig. 14. Input voltage.

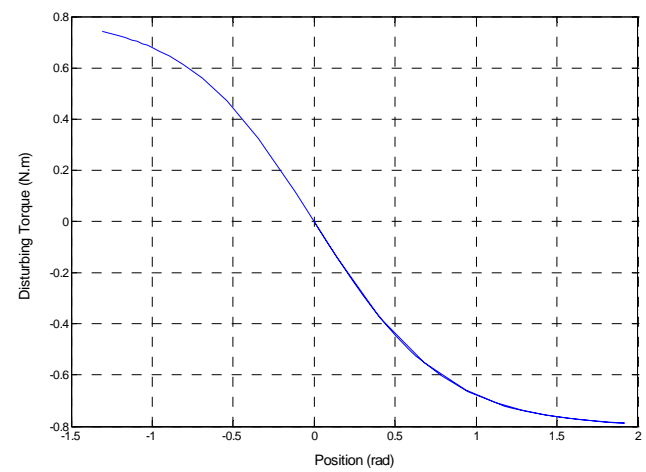

Fig. 15. Backlash disturbing torque.

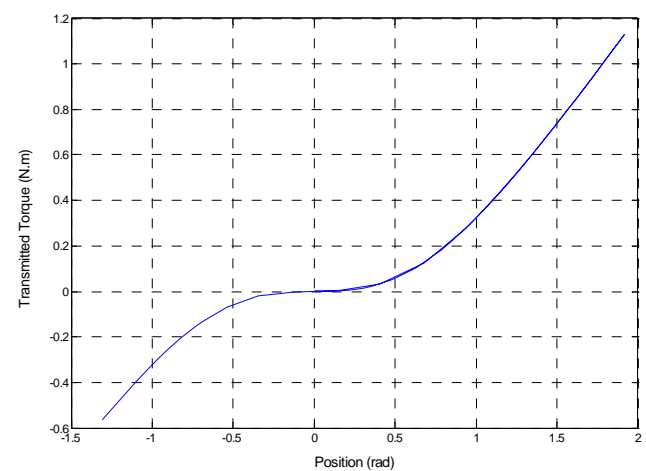

Fig. 16. Transmitted torque via a dead zone and flexible link.

Relation (ARR) along with the corresponding Fault Signature Matrix (FSM) can be generated. These tools allow to detect and isolate the possible faults present on the physical system. Note that for this application, and comparing with the existing model based FDI methods where the faults are not modelled; in the proposed contribution the backlash is considered as a fault and taken into account in the system dynamic model. So, its related variables appear in the generated ARR and its dynamics can be simulated through the ARR equations by using an appropriate software. 
The main steps to generate the list of ARR and the FSM by using the method developed in [4] are summarized bellow:

- Build the bond graph model in preferred integral causality.

- Put the bond graph model in preferred derivative causality (with sensor causality inversion if necessary).

- Write the constitutive relation for each junction.

- Eliminate the unknown variables from each constitutive relation by covering the causal paths on the bond graph model.

- Generate the list of ARR and the corresponding FSM.

Note that for an observable system, with none unresolved algebraic loops, the number of ARR generated is equal to the number of detectors on the bond graph model [4].

The bond graph model, in derivative causality, of the system under study is shown in Fig. 17.

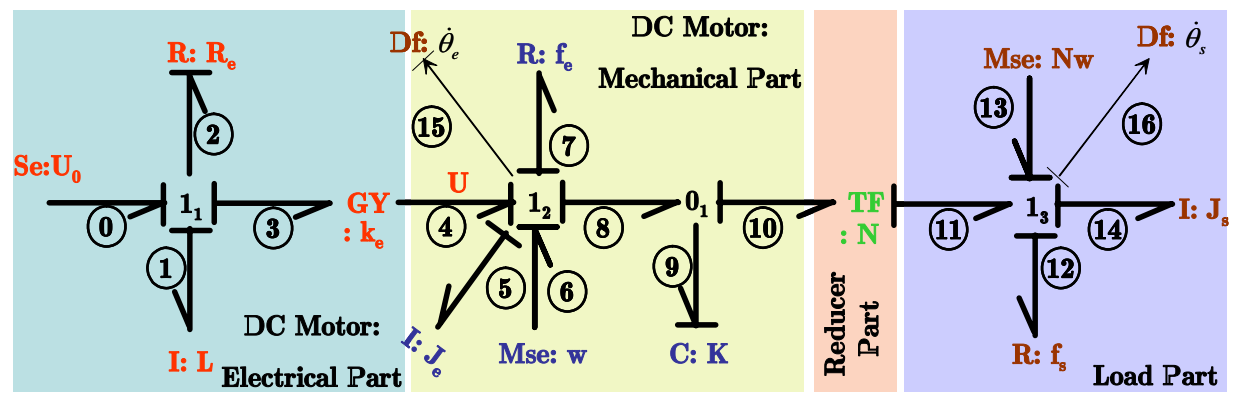

Fig. 17. Bond graph model in derivative causality of the system.

The constitutive relations of junctions ' $1_{1}$ ', ' $1_{2}$ ', ' $0_{1}$ ' and ' $1_{3}$ ' are given by the following equations:

$$
\begin{aligned}
e_{2} & =e_{0}-e_{1}-e_{3}, \\
e_{15} & =e_{4}-e_{5}+e_{6}-e_{7}-e_{8}, \\
f_{10} & =f_{8}-f_{9}, \\
e_{11} & =e_{12}+e_{14}+e_{16}-e_{13} .
\end{aligned}
$$

Two structurally independent ARR can be generated from Eqs. (14) and (15) after eliminating the unknown variables. This is because the system is structurally observable and does not contain any algebraic loop. The unknown variables elimination process is achieved by following the causal paths, from known to unknown variables, on the bond graph model.

For Eq. (14) the unknown variables $e_{15}, e_{5}, e_{6}, e_{7}$ and $e_{8}$ can be calculated as follows: 


$$
\left\{\begin{array}{l}
e_{15}=0 \\
e_{5}=J_{e} \cdot \frac{d f_{5}}{d t}=J_{e} \cdot \frac{d \dot{\theta}_{e}}{d t} \\
e_{6}=w \\
e_{7}=f_{e} \cdot f_{7}=f_{e} \cdot \dot{\theta}_{e} \\
e_{8}=e_{10}=\frac{1}{N} \cdot e_{11}=\frac{1}{N} \cdot\left(f_{s} \cdot \dot{\theta}_{s}-N \cdot w+J_{s} \cdot \frac{d \dot{\theta}_{s}}{d t}\right) .
\end{array}\right.
$$

Once the variable $e_{4}$ is determined, Eq. (14) can be written only in terms of known variables which is in fact an ARR. Eq. (13) can be rewritten as:

$$
e_{3}=U_{0}-e_{2}-e_{1}=U_{0}-R_{e} \cdot f_{3}-L \cdot \frac{d f_{3}}{d t}
$$

with

$$
\left\{\begin{array}{l}
f_{3}=\frac{1}{k_{e}} \cdot e_{4} \\
e_{4}=J_{e} \cdot \frac{d \dot{\theta}_{e}}{d t}-w+f_{e} \cdot \dot{\theta}_{e}+\frac{1}{N} \cdot\left(f_{s} \cdot \dot{\theta}_{s}-N \cdot w+J_{s} \cdot \frac{d \dot{\theta}_{s}}{d t}\right) \\
e_{3}=k_{e} \cdot f_{4}=k_{e} \cdot \dot{\theta}_{e} .
\end{array}\right.
$$

Then Eq. (18) becomes

$$
k_{e} \cdot \dot{\theta}_{e}=U_{0}-\frac{R_{e}}{k_{e}} \cdot e_{4}-\frac{L}{k_{e}} \cdot \frac{d e_{4}}{d t} .
$$

The following first ARR is then deduced by replacing the expression of $e_{4}$, calculated from Eq. (19), in Eq. (20):

$$
\begin{aligned}
A R R_{1} & : k_{e} \cdot \dot{\theta}_{e}-U_{0}+\frac{R_{e}}{k_{e}} \cdot\left(J_{e} \cdot \frac{d \dot{\theta}_{e}}{d t}-w+f_{e} \cdot \dot{\theta}_{e}+\frac{1}{N}\left(f_{s} \cdot \dot{\theta}_{s}-N \cdot w+J_{s} \cdot \frac{d \dot{\theta}_{s}}{d t}\right)\right) \\
& +\frac{L}{k_{e}} \cdot \frac{d}{d t}\left(J_{e} \cdot \frac{d \dot{\theta}_{e}}{d t}-w+f_{e} \cdot \dot{\theta}_{e}+\frac{1}{N}\left(f_{s} \cdot \dot{\theta}_{s}-N \cdot w+J_{s} \cdot \frac{d \dot{\theta}_{s}}{d t}\right)\right)=0 .
\end{aligned}
$$

The second ARR can be deduced from equation 15 . The unknown variables $f_{10}, f_{8}$ and $f_{9}$ of this equation can be calculated from the known ones as follows: 


$$
\left\{\begin{array}{l}
f_{10}=N \cdot f_{11}=N \cdot \dot{\theta}_{s} \\
f_{8}=f_{15}=\dot{\theta}_{e} \\
f_{9}=\frac{1}{K} \cdot \frac{d e_{9}}{d t}=\frac{1}{K} \cdot N \cdot \frac{d}{d t}\left(e_{12}+e_{14}+e_{16}-e_{13}\right)
\end{array}\right.
$$

where

$$
\left\{\begin{array}{l}
e_{13}=N \cdot w, \\
e_{12}=f_{s} \cdot f_{12}=f_{s} \cdot \dot{\theta}_{s}, \\
e_{14}=J_{s} \cdot \frac{d f_{14}}{d t}=J_{s} \cdot \frac{d \dot{\theta}_{s}}{d t}, \\
e_{16}=0 .
\end{array}\right.
$$

The second ARR is then deduced by replacing the unknown variables of Eqs. (21) and (22) in Eq. (15):

$$
A R R_{2}: N \cdot \frac{d}{d t}\left(f_{s} \cdot \dot{\theta}_{s}+J_{s} \cdot \frac{d \dot{\theta}_{s}}{d t}-N \cdot w\right)+K \cdot\left(N \cdot \dot{\theta}_{s}-\dot{\theta}_{e}\right)=0
$$

Once the list of ARR is generated, the FSM can be built by deducing the signatures of the system components on each residual. Note that each component in the physical system can be represented by one or more variable (or parameters) in the residuals. A residual $r_{i}$ is a numerical evaluation of an $A R R_{i}$. The corresponding residuals $r_{1}$ and $r_{2}$ of $A R R_{1}$ and $A R R_{2}$ are given by the following relations:

$$
\begin{aligned}
r_{1}= & k_{e} \cdot \dot{\theta}_{e}-U_{0}+\frac{R_{e}}{k_{e}} \cdot\left(J_{e} \cdot \frac{d \dot{\theta}_{e}}{d t}-w+f_{e} \cdot \dot{\theta}_{e}+\frac{1}{N}\left(f_{s} \cdot \dot{\theta}_{s}-N \cdot w+J_{s} \cdot \frac{d \dot{\theta}_{s}}{d t}\right)\right) \\
& +\frac{L}{k_{e}} \cdot \frac{d}{d t}\left(J_{e} \cdot \frac{d \dot{\theta}_{e}}{d t}-w+f_{e} \cdot \dot{\theta}_{e}+\frac{1}{N}\left(f_{s} \cdot \dot{\theta}_{s}-N \cdot w+J_{s} \cdot \frac{d \dot{\theta}_{s}}{d t}\right)\right), \\
r_{2}= & N \cdot \frac{d}{d t}\left(f_{s} \cdot \dot{\theta}_{s}+J_{s} \cdot \frac{d \dot{\theta}_{s}}{d t}-N \cdot w\right)+K \cdot\left(N \cdot \dot{\theta}_{s}-\dot{\theta}_{e}\right) .
\end{aligned}
$$

The corresponding FSM of the system under study is given in table 2 .

In this table 2, the rows represent the components signatures and the columns are respectively fault detectability $D_{b}$, fault isolability $I_{b}$, and first and second residual $r_{1}$ and $r_{2}$. A ' 1 ' value on respectively $D_{b}$ and $I_{b}$ columns means that 
Table 2

\begin{tabular}{|l|ccc|cc|}
\hline & & $D_{b}$ & $I_{b}$ & $r_{1}$ & $r_{2}$ \\
\hline Input velocity sensor & $\dot{\theta}_{e}$ & 1 & 0 & 1 & 1 \\
Output velocity sensor & $\dot{\theta}_{s}$ & 1 & 0 & 1 & 1 \\
Electrical part & $L$ & 1 & 0 & 1 & 0 \\
Mechanical part & $R$ & 1 & 0 & 1 & 0 \\
Disturbing torque & $J_{e}$ & 1 & 0 & 1 & 0 \\
Load part & $f_{e}$ & 1 & 0 & 1 & 0 \\
& $w$ & 1 & 0 & 1 & 1 \\
& $J_{s}$ & 1 & 0 & 1 & 1 \\
& $f_{s}$ & 1 & 0 & 1 & 1 \\
\hline
\end{tabular}

Fault signature matrix of the electromechanical system.

faults on the corresponding components are detectable and isolable. The presence of ' 1 ' value on $r_{1}$ and $r_{2}$ columns shows the influence of the corresponding components on the residual dynamics.

In this application, the FSM helps to detect the presence of backlash phenomenon issued from a possible mechanical wear.

\section{Simulation Results}

Simulation tests have been done on backlash system model of Fig. 1, where inertia, friction and dead zone parameters have been identified in [6] and given in table 1. These simulation tests allow to show how backlash phenomenon can be detected for typical electromechanical system of Fig. 1, by using the FDI algorithm described before. The software tool used for this simulation is $S Y M B O L S 2000$ to generate the ARRs and their corresponding residuals in symbolic form, then Matlab - Simulink for numerical results. Tests have been done with an open loop scheme, where only input and output positions of reducer part are experimentally measured. Note that for the studied test bench system, the dead zone magnitude could be varied until 0,2 rad. So, if this magnitude is maintained with the elasticity characteristic given in table 1 , the estimated disturbing torque $w$ will describe the sigmoid curve of Fig. 18 and expressed in Eq. (3).

As shown in Fig. 19(c) and Fig. 19(d), while the input reducer axis rotates at around $5,2 \mathrm{rad} / \mathrm{s}$, the input one goes to $1,7 \mathrm{rad} / \mathrm{s}$; where each axis follows successively trajectories of Fig. 19(a) and Fig. 19(b). For this slow motion, 


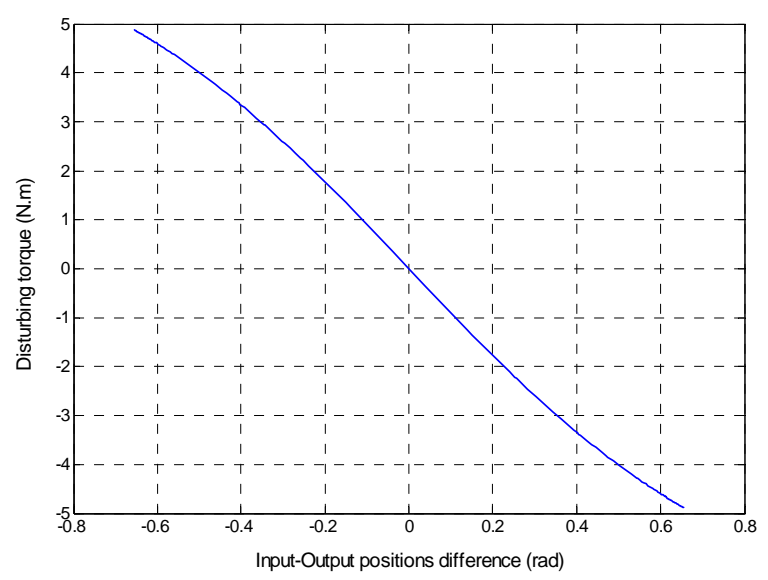

Fig. 18. Disturbing torque evolution.

one needs to detect the fault issued from backlash phenomenon in order to illustrate its impact on nominal system operating. In this case, mechanical transmission inside the reducer part of the system given in Fig. (1) is represented by a flexible link and a dead zone area varying between 0 and 0,2 rad.

Two distinct detections of dead zone are presented: the first one during temporal interval $[7,5 ; 12] s$, where the disturbing torque $w$ of Fig. 20(b) becomes non null and varies from 0 to $-4,75 \mathrm{~N} . \mathrm{m}$. The disturbing torque $w$ is in linear dependence of $j_{0}$ (see Eq. (3)), so its variation out of zero value, shows the presence of dead zone. This latter varies within $[0 ; 0,18] \mathrm{rad}$ (Fig. 20(a)) and is noticed via evolution of $A R R_{1}$ and $A R R_{2}$ (Fig. 20(c) and Fig. 20(d)). The second detection is given between $[17 ; 21] s$, where input and output positions and velocities of reducer part given in Fig. 19(a), Fig. 19(b), Fig. 19(c) and Fig. 19(d) are affected. This second detection presented in $A R R_{1}$ and $A R R_{2}$ (Fig. 20(c) and Fig. 20(d)), by a positive residual values, shows an increase of disturbing torque (Fig. 20(b)) until zero value corresponding to position gap (Fig. 20(a)) due to flexible link of $1 \mathrm{rad}$. 

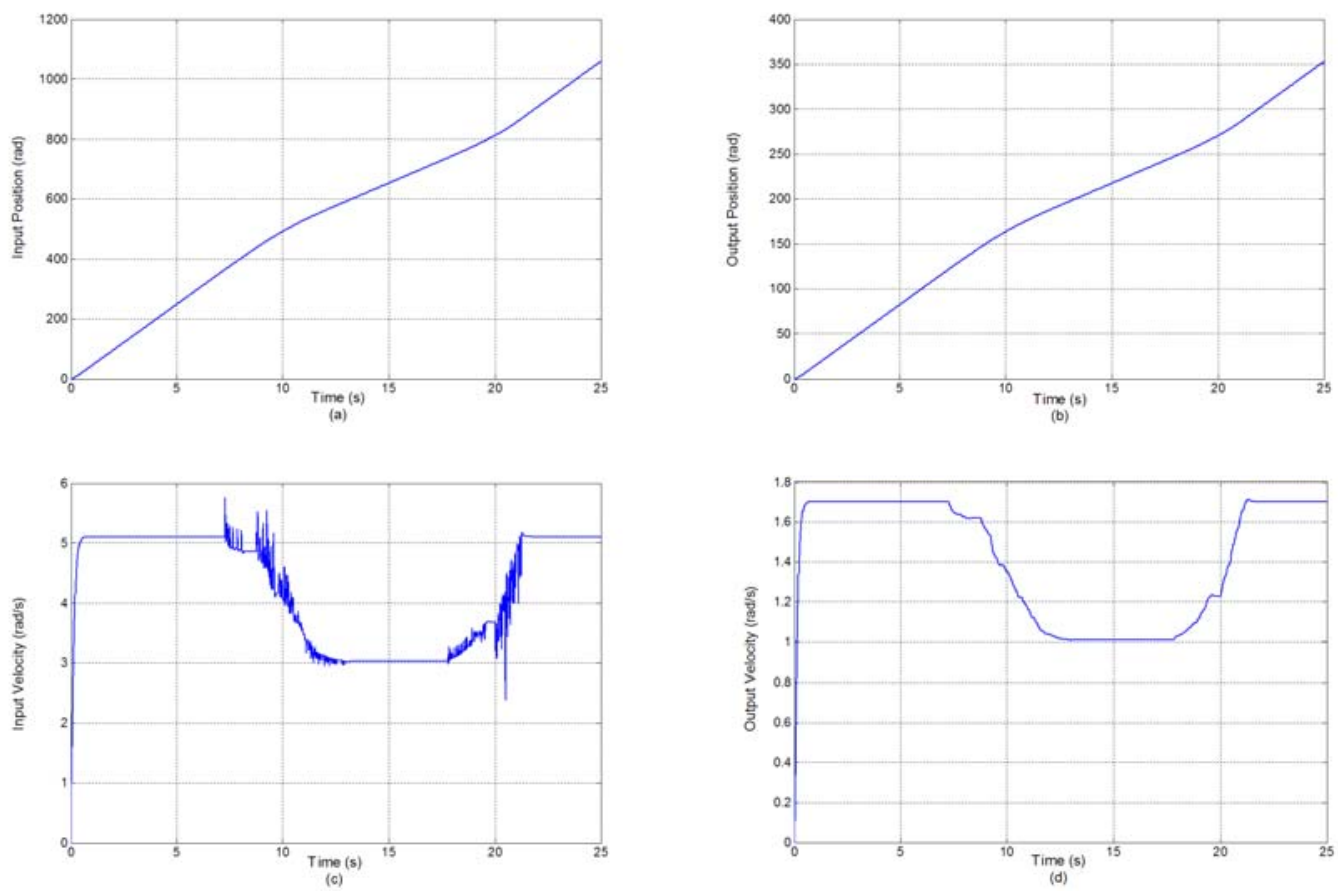

Fig. 19. (a) Input reducer part position, (b) output reducer part position, (c) Input reducer part velocity and (d) output reducer part velocity.
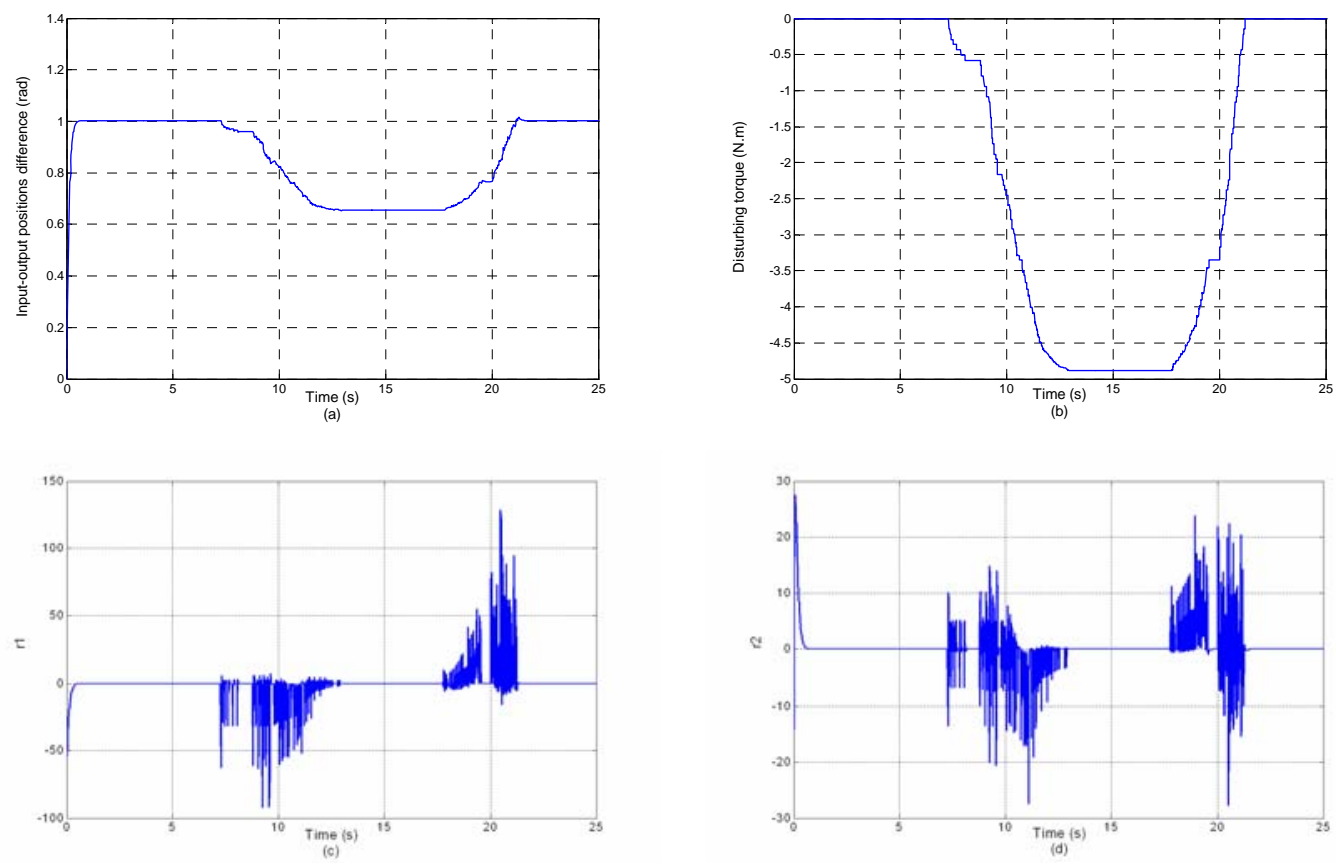

Fig. 20. (a) Temporal evolution of input-output reducer position gap with presence of dead zone, (b) temporal evolution of disturbing torque with dead zone variation, (c) dead zone variation acting on first ARR and (d) dead zone variation acting on second ARR. 


\begin{tabular}{|c|c||c|c|}
\hline Parameters & N. Values & Parameters & N. Values \\
\hline$J_{m}$ & $0.01\left({\left.\mathrm{Kg} . \mathrm{m}^{2}\right)}^{2}\right.$ & $N$ & 1 \\
\hline$f_{m}$ & $0.015(\mathrm{~N} . \mathrm{m} . \mathrm{sec} / \mathrm{rad})$ & $j_{0}$ & $0.1-0.8(\mathrm{rad})$ \\
\hline$J_{s}$ & $0.00002\left(\mathrm{Kg} . \mathrm{m}^{2}\right)$ & $L$ & $1.9(\mathrm{H})$ \\
\hline$f_{s}$ & $0.02(\mathrm{~N} . \mathrm{m} . \mathrm{sec} / \mathrm{rad})$ & $R_{e}$ & $7.6(\Omega)$ \\
\hline
\end{tabular}

Table 3

System parameters.

\section{Experimental Results}

Experimental results have been done on system of (Fig. 1), where the system parameters are given in table 3 . These parameters are identified off-line on the real system, using structural and analytical identification methods. A PD controller is applied on the real system with the coefficients $K_{P}=1.25$ and $K_{D}=0.5$.

At the fist step, the dead zone between the two independent mechanisms of the load part are mechanically eliminated (see Fig. 1). Then, control signal of Fig. (21-a) is applied, where input and output position signals are superposed in Fig. (21-b). The difference between the two positions is given by Fig. (21-c). Normally, the dead zone is eliminated mechanically, but it is already present as it is shown in Fig. (21-d). This is due to the presence of initial dead zone which is not considered in the modelling and practically is located between transmitted gears and useful for the initial transmission. This initial dead zone is taken as the tolerant value that we admit for the backlash phenomenon. The residual signals $r_{1}$ and $r_{2}$ in presence of this initial values are given successively Fig. (21-e) and Fig. (21-f).

At the second step, we omitted the screw which link the two mechanisms of the load part (see Fig. 1). Then, an important dead zone area is introduced. For the same control signal as presented in step one, it is applied again for the second step (see Fig. (22-a)), where the backlash effect is considerable according to the position signals and their difference in Fig. (21-b) and Fig. (21-c). The hysteresis characteristic between the positions, issued from the presence of an important dead zone is illustrated in Fig. (21-d). Finally, residual signals, which show the increase of their amplitude comparing to the step1 are given in Fig. (21-e) and Fig. (21-f). The asymmetric allure of the signals is due to the initial position of the two mechanisms which is not confounds with the middle of the dead zone. 

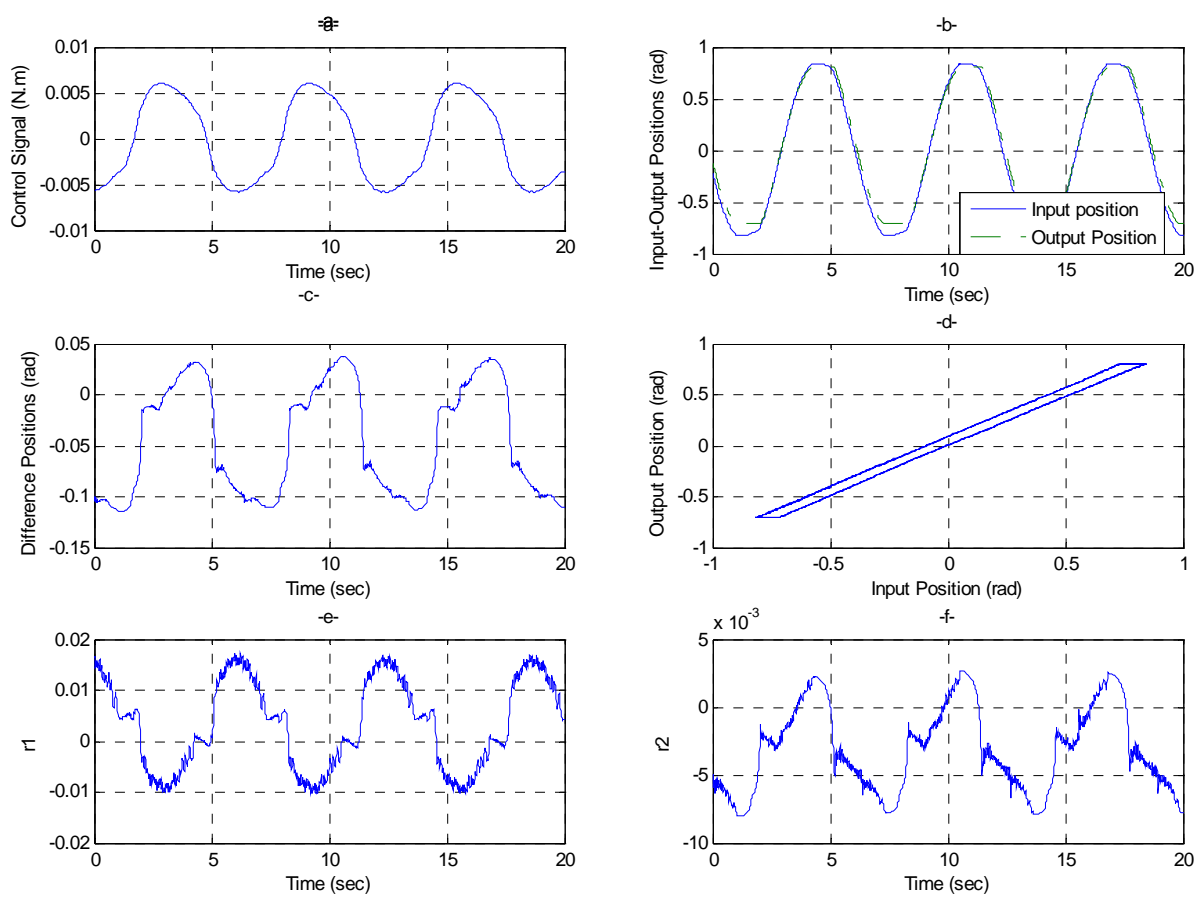

Fig. 21. Experiments with initial and significant backlash phenomenon: (a) Input Control Signal, (b) Input-Output Reducer Part, (c) Input-Output Difference Position, (d) Input-Output Hysteresis Behavior, (e) Residual Signal 1, (f) Residual Signal 2.

\section{Conclusion}

In this work, a model based FDI application on electromechanical system is presented using bond graph approach. At first, the bond graph, in preferred integral causality, is used to model the nonlinear electromechanical system, by combining the multi-physics dynamics. Then, this bond graph tool is used to generate the residuals via the analytical redundancy relations, in order to detect the presence of perturbed backlash phenomenon. Finally, the fault signature matrix is obtained from the residuals expressions, by putting the bond graph model of the system in preferred derivative causality. These fault indicators allowed to monitor the physical system, and to distinguish the undesirable backlash from the useful one by observing the residual responses as shown by the simulation and the experimental results. 

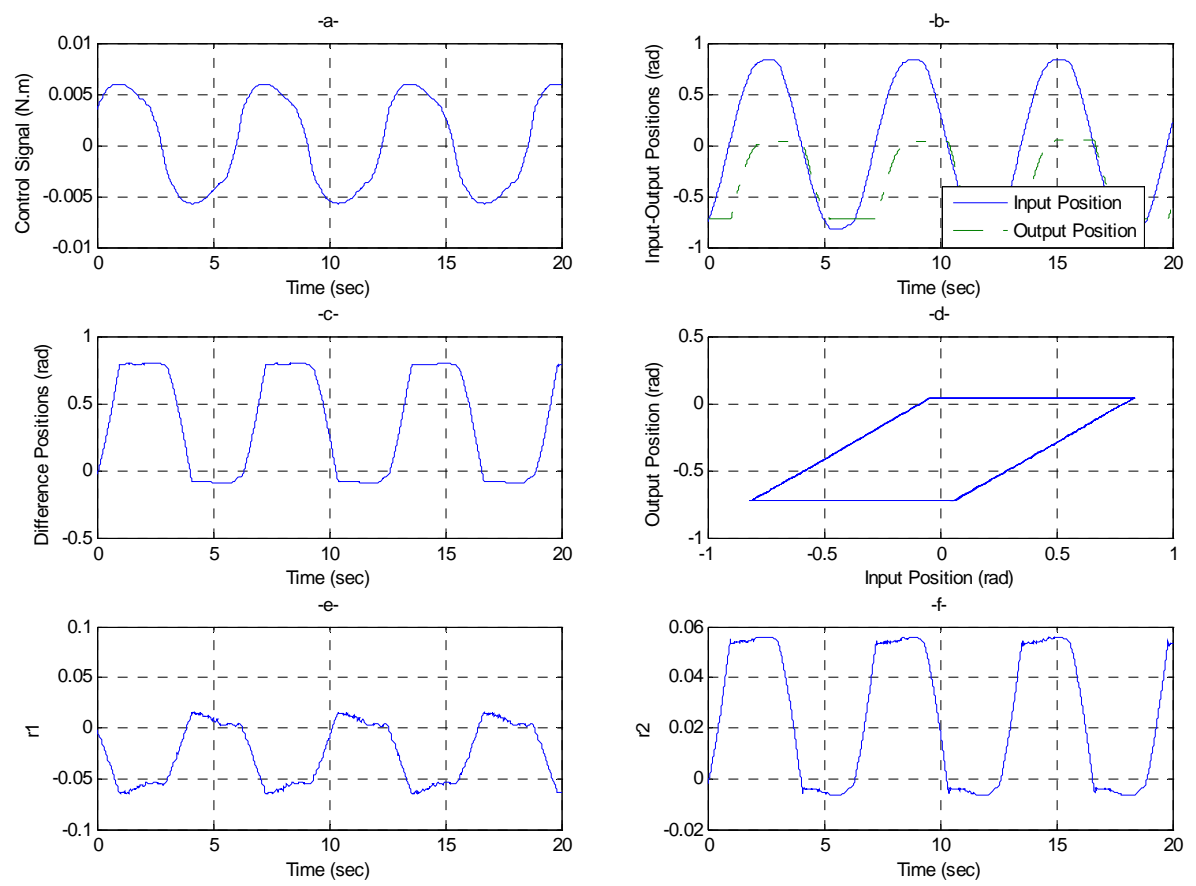

Fig. 22. Experiments with important backlash fault: (a) Input Control Signal, (b) Input-Output Reducer Part, (c) Input-Output Difference Position, (d) Input-Output Hysteresis Behavior, (e) Residual Signal 1, (f) Residual Signal 2. 


\section{References}

[1] B. Ould Bouamama, A.K. Samantaray, M. Staroswiecki, and G. DauphinTanguy. Derivation of constraint relations from bond graph models for fault detection and isolation. In International Conference on Bond Graph Modeling and Simulation (ICBGM'03), pages 104-109. Simulation Series Vol.35, No.2, ISBN 1-56555-257-1, 2003.

[2] G. Dauphin-Tanguy, A. Rahmani, and C. Sueur. Bond graph aided design of controlled systems. Simulation Practice and Theory, 7(5-6):493 - 513, 1999.

[3] M. A. Djeziri, R. Merzouki, B. Ould Bouamama, G. Dauphin Tanguy. Fault detection of backlash phenomenon in mechatronic system with parameter uncertainties using bond graph approach. In International Conference on Mechatronics and Automation (ICMA'06), pages 600-605, Luoyang, China, June 25 - 282006 .

[4] B. Ould Bouamama, K. Medjaher, A. K. Samantaray, and M. Staroswiecki. Supervision of an industrial steam generator. part I: Bond graph modelling. Control Engineering Practice, 2005. In press, available online at www.sciencedirect.com.

[5] R. Isermann. Supervision, fault detection and fault diagnosis methods - an introduction. Control Engineering Practice, Vol. 5, No. 5:639 - 652, 1997.

[6] R. Merzouki, J. C. Cadiou, and N. K. M'Sirdi. Compensation of friction and backlash effects in an electrical actuator. Journal of Systems and Control Engineering, 218(12):75—84, March 2004.

[7] R. Merzouki and J. C. Cadiou. Estimation of backlash phenomenon in the electromechanical actuator. Control Engineering Practice, 13(8):973-983, August 2005.

[8] R. Merzouki, J. A. Davila Montoya, L. M. Fridman and J. C. Cadiou. Backlash phenomenon observation and identification in electromechanical system. Control Engineering Practice, Vol 15/4, pp 447-457, 2007.

[9] T. Murakami and N. Nakajima. Compter-aided design-diagnosis using feature description. In J.S. Gero, editor, Artificial Intelligence in Engineering: Diagnosis and Learning, pages 199-226. Elsevier, 1988.

[10] H.M. Paynter. Analysis and design of Engineering Systems. M.I.T. Press, 1961.

[11] M. Staroswiecki and G. Comtet-Varga. Analytical redundancy relations for fault detection and isolation in algebraic dynamic systems. Automatica, 37:687 -699, 2001.

[12] A. Mukherjee and A.K. Samantaray. System modelling through bond graph objects on SYMBOLS 2000. In International Conference on Bond Graph Modeling and Simulation (ICBGM'01), pages 164-170. Simulation Series, Vol. 33, No. 1, ISBN 1-56555-103-6, 2001. 
[13] J. A. Tenreiro Machado. Variable structure control of manipulators with joints having flexibility and backlash. Journal Systems Analysis-ModellingSimulation, 23(0):93-101, 1996.

[14] G. Tao and P.V. Kokotovic. Contiuous-time adaptive control of systems with unknown backlash. IEEE Transactions on Automatic Control, 40(2):10831087, 1995.

[15] V. Venkatasubramanian, R. Rengaswamy, and S.N. Kavuri. A review of process fault detection and diagnosis. Part II: Qualitative models and search strategies. Computers and Chemical Engineering, 27:313-326, 2003. 